\title{
Conflict over fertilization underlies the transient evolution of reinforcement
}

\author{
Catherine A. Rushworth ${ }^{1,2}$, Alison M. Wardlaw ${ }^{1}$, Jeffrey Ross-Ibarra ${ }^{2,3}$ and Yaniv Brandvain ${ }^{1, *}$ \\ ${ }^{1}$ Dept. of Plant and Microbial Biology, University of Minnesota, St. Paul, MN, USA, ${ }^{2}$ Dept. of Evolution and Ecology and Center for Population Biology, \\ University of California, Davis, CA, USA, ${ }^{3}$ Genome Center, University of California, Davis, CA, USA
}

\begin{abstract}
When two populations or species hybridize, their offspring often experience reductions in fitness relative to either parental population. The production of low fitness hybrids may be prevented by the evolution of increased prezygotic isolation; a process known as reinforcement. Theoretical challenges to the evolution of reinforcement are generally cast as a coordination problem - e.g., linkage disequilibrium between trait and preference loci is difficult to maintain in the face of recombination. However, the evolution of reinforcement also poses a potential conflict between mates. For example, the opportunity costs to hybridization may differ between the sexes or species. This is particularly likely for postmating prezygotic isolation, as the ability to fertilize both conspecific and heterospecific eggs is beneficial to male gametes, but heterospecific mating may incur a cost for female gametes. Motivated by this problem, we develop a population genetic model of interspecific conflict over reinforcement, inspired by 'gametophytic factors', which act as postmating prezygotic barriers among Zea mays subspecies. We demonstrate that this conflict results in the transient evolution of reinforcement - after female preference for a conspecific gamete trait rises to high frequency, male traits adaptively introgress into the other population. Ultimately the male gamete trait fixes in both species, and prezygotic isolation returns to pre-reinforcement levels. We interpret geographic patterns of isolation among $Z$. mays subspecies in light of these findings, and suggest when and how this conflict can be mediated. Our results suggest that sexual conflict may pose an understudied obstacle to the evolution of reinforcement via postmating prezygotic isolation.
\end{abstract}

KEYWORDS Reinforcement, Sexual conflict, Speciation, Hybridization

\section{Introduction}

In the highlands of Mexico, Zea mays mexicana, a wild teosinte, often grows alongside and hybridizes with its domesticated relative Z. m. mays (hereafter, maize), at a rate of approximately $1 \%$ per generation (Ellstrand et al. 2007). Maize-teosinte hybrids are often disfavored - in farm fields, cultivation and retention of hybrids is almost never desirable, leading to selection via anthropogenic weeding, while maize traits expressed in teosinte environments are likely to be maladapted (Wilkes 1977; Ellstrand et al. 2007). The relatively frequent production of low fitness hybrids should favor the adaptive evolution of enhanced reproductive isolation between these subspecies, minimizing the reproductive effort wasted in producing low-fitness hybrids in a process known as reinforcement (Dobzhansky 1937; Servedio and Noor 2003). In fact, there is some evidence that teosinte shows elevated pollen discrimination - three separate stylarexpressed loci underlie preferential fertilization by teosinte-like pollen (Moran Lauter et al. 2017; Lu et al. 2014, 2019; Wang et al. 2018; Lu et al. 2020). Despite effectively rejecting pollen from allopatric maize, these barriers are ineffective with pollen from sympatric maize (Kermicle et al. 2006). We develop population genetic theory to address this conundrum - what evolution-

* Dept. of Plant and Microbial Biology, University of Minnesota, St. Paul, MN, USA E-mail: ybrandva@umn.edu ary processes have created this pattern where teosinte rejects maize pollen from allopatric but not sympatric populations? Our results suggest that tensions between the sexes could limit the reinforcement of postmating prezygotic isolating barriers in maize, and are applicable to numerous other systems.

Theory shows that reinforcement is plausible (Liou and Price 1994; Servedio and Kirkpatrick 1997), and lab experiments show that it can evolve (e.g. Matute 2010). In nature, correlative studies suggest reinforcement does occur (e.g. Coyne and Orr 1989). These correlative studies compare the extent of prezygotic isolation (or some proxy for it, such as male trait divergence) between allopatric and sympatric populations of the same species pair, under the assumption that reinforcement can result in the elevation of reproductive isolation in sympatry, but not in allopatry. Most notably, Coyne and Orr $(1989,1997)$ documented enhanced behavioral isolation of sympatric Drosophila species, and similar observations have been found in numerous animals (reviewed in Coyne and Orr 2004) and plants (Hopkins 2013). As such, the surprising pattern of reproductive isolation between maize and teosinte in the Mexican highlands cannot simply be explained as the implausibility of reinforcement.

To date, most theoretical, comparative, and experimental studies of reinforcement have focused on the evolution of enhanced premating isolation. However, it has long been argued that natural selection could favor increased isolation at any point in development (Wallace 1889). For example, Coyne 
(1974) and Johnson and Wade (1995) argue that a reinforcementlike process could favor various forms of hybrid inviability if it enhanced maternal inclusive fitness - an argument consistent with patterns of elevated early inviability in sympatric $\mathrm{Neu}$ rospora species (Turner et al. 2010). Likewise, Turissini et al. (2017) explored the possibility of reinforcement of postmating prezygotic barriers. Thus, although some authors have argued on historical grounds to restrict the term of reinforcement to enhanced prezygotic isolation (e.g. Johnson 2008), adaptive evolution of enhanced isolation (hereafter reinforcement) can theoretically occur before or after mating.

Nonetheless, "bona fide cases of reinforcement and of gametic isolation are still rare" (Turissini et al. 2017). Previously claimed cases of the reinforcement of postmating prezygotic barriers in sea urchins (Lessios 2007; Geyer and Palumbi 2003; Zigler et al. 2003), and mussels (Slaughter et al. 2008) seem to reflect barriers that are ineffective (Slaughter et al. 2008) and/or reflect the action of selection on intraspecific matings (Geyer and Palumbi 2003), rather than the reinforcement of reproductive isolation. In fact, we know of only a handful of cases of gametic (a.k.a. postmating prezygotic) isolation. One such case is in plants- where Kay (2006) found that Costus scaber, preferentially rejects $C$. pulverulentus pollen when the populations are sympatric, but not when they are allopatric (Kay and Schemske 2008). Additionally, data from three Drosophila species pairs suggest the reinforcement of postmating prezygotic isolation (Matute 2010; Castillo and Moyle 2019; Poikela et al. 2019). Counter to these case studies, broader comparative surveys in both Drosophila (Turissini et al. 2017) and plants (Moyle et al. 2004) fail to find a broad signature of reinforcement of postmating prezygotic barriers. Thus, while the reinforcement of postmating prezygotic isolation may be more difficult to observe than more obvious color or behavioral differences (Turissini et al. 2017), the absence of broad correlative patterns in explicit empirical tests suggest that the paucity of cases of postmating prezygotic isolation is not entirely attributable to difficulties in observing it.

This meager empirical evidence for the reinforcement of postmating prezygotic barriers contradicts intuition that this would be common in sessile organisms (e.g. plants and mussels), as this is their only direct control over mate choice. Instead, reinforcement in such systems generally involves premating barriers such as temporal changes in mating (e.g. Paterniani 1969; Clifton 1997), or changes in pollinator attraction (see Hopkins 2013, for a review of all known cases of reinforcement in plants). Why are there so few clear-cut cases of the reinforcement of postmating prezygotic isolation? We investigate this question by developing a population genetic model, inspired by the biology of pollen-pistil incompatibilities, in hybridizing subspecies of Zea mays. We note that while these incompatibilities tend to isolate maize and teosinte, they are variable across Zea mays subspecies, and were first identified in crosses between maize lines (Mangelsdorf and Jones 1926).

Our model suggests that sexual conflict could explain the patterns of postmating prezygotic isolation between maize subspecies, and the paucity of evidence for the reinforcement of postmating prezygotic isolation. Reinforcement is generally conceptualized as the evolution of enhanced prezygotic isolation at a locus underlying assortative mating (e.g. Felsenstein 1981) via female preference or trait matching (Kopp et al. 2018). These mechanisms assume either that males and females have a shared interest in avoiding the production of

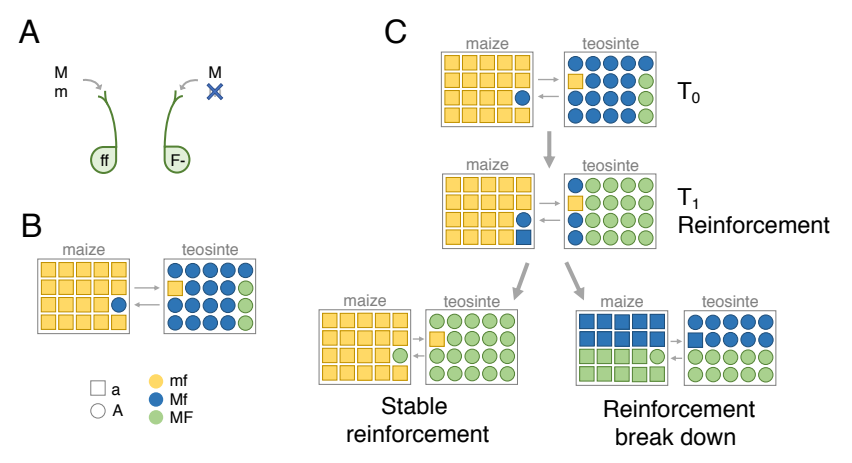

Figure 1 Model dynamics (A) Gametophytic factors of maize and teosinte - the dominant $F$ allele encodes a stylar barrier to mating that can only be overcome by the pollen-expressed compatibility allele M. (B) Pollen flows between two diverged populations (hereafter maize and teosinte), facing different selective pressures. Colors denote gametophyic factor haplotype, while shapes signify genotypes at a divergently selected allele. Initially, maize is fixed for the non-barrier style-expressed allele and the incompatible pollen-expressed allele, as well as a locally adaptive allele at locus A (haplotype $m f a$ ). Teosinte is initially fixed for the male compatibility allele and a second locally adapted allele, and has low to intermediate frequency of the stylar barrier allele (haplotypes MfA and MFA). (C) We run the model from this initial parameterization at generation, $T_{0}$, until $F$ allele reaches is equilibrium frequency in the bottom panel. Some reinforcement evolves by time $T_{1}$ : the $F$ allele is increasing in frequency in teosinte, resulting in postmating prezygotic isolation between populations. At this point, two outcomes are possible: the $F$ allele may fix in the teosinte population despite gene flow, leading to complete reinforcement, or the $M$ allele may fix in both populations, leading to the breakdown of reinforcement.

maladapted hybrids, or that preventing the production of lowfitness hybrid offspring is beneficial to females and presents an opportunity cost to males. However, reproductive interactions are often more fractious than this - the costs of reproductive effort can differ by sex and /or reproductive stage (Arnqvist and Rowe 2005). Because male and female interests are not always aligned, sexual conflict over the hybridization rate (Parker and Partridge 1998) may create an often overlooked hurdle to the evolution of reinforcement. We show that asymmetry in costs and benefits of hybridization faced by males and females results in the transient evolution of reinforcement, leaving footprints consistent with observations in Z. mays subspecies. Our results are relevant to many potential cases of reinforcement by gamete recognition in plants, broadcast spawners (e.g. Lysin/VERL in abalone (Swanson and Vacquier 1998) or Bindin/EBR1 (Metz et al. 1994) in sea urchins), and even cases of internal fertilization in which premating isolation is inefficient, costly, or otherwise unpreferred (Turissini et al. 2017).

\section{Results}

\section{Model overview}

Model set up: Our model is inspired by empirical observations in teosinte, a wild subspecies of the grass Zea mays, where pairs of tightly-linked loci - termed gametophytic factors - medi- 
ate postmating prezygotic isolation with domesticated maize (Moran Lauter et al. 2017; Lu et al. 2014, 2019; Wang et al. 2018). We therefore refer to maize and teosinte in our model to simplify biological interpretation. We model a single gametophytic factor as a gametic incompatibility (sensu Lorch and Servedio 2007) made up of two tightly linked loci. The stylar-acting locus, $\mathcal{F}$, is under diploid control. We assume the incompatibility is dominant - that is, styles with one or two $F$ alleles discriminates between pollen genotypes, preferentially mating with $M$ (Figure 1A), while styles homozygous for the compatability allele, $f$ permit fertilization by pollen with any haploid genotype at the $\mathcal{M}$ locus. We note that this notation differs from that of the existing literature on gametophytic factors (see Methods). We assume a 'two-island model', with all migration occurring in pollen, and model isolation by local adaptation (following Kirkpatrick 2001) with n locally adapted loci, each noted as $\mathcal{A}_{i}$, where the subscript $i$ is an arbitrary index of loci. We model viability selection at the diploid stage, with multiplicative fitness within and among loci.

Model Parameters: We assume selection coefficients $s_{\text {maize }}$ and $s_{\text {teo }}$ in maize and teosinte environments, respectively. Every generation, $g_{\text {maize } \rightarrow \text { teo }}$ of the teosinte environment pollen pool originates from plants in the maize environment, and $g_{\text {teo } \rightarrow \text { maize }}$ of pollen in the maize environment originates from plants in the teosinte environment (Figure 1B). We assume a marker order of $\mathcal{A}_{1} \mathcal{M F}$, with recombination rates $r_{\mathcal{A}_{1} \mathcal{M}}$ and $r_{\mathcal{M F}}$ and that locally adaptive loci $\mathcal{A}_{2}$ through $\mathcal{A}_{n}$ are unlinked to one another and the $\mathcal{M F}$ loci. Thus, we modify the strength and symmetry of selection and migration, the number of loci underlying local adaptation, and the recombination rates across the $\mathcal{A}_{1} \mathcal{M} \mathcal{F}$ haplotype.

To highlight the evolutionary dynamics of this system, we first focus on a single set of parameter values $\left(s_{\text {teo }}=s_{\text {maize }}=\right.$ 0.75 , symmetric migration of $0.1\left(g_{\text {maize } \rightarrow \text { teo }}=g_{\text {teo } \rightarrow \text { maize }}=\right.$ 0.1 , and recombination rate $r_{\mathcal{A M}}=r_{\mathcal{M F}}=0.0001$, consistent with the low recombination rate between male and female factors in Lu et al. (2014). $M$ is initially fixed in teosinte and absent in maize, and the $F$ allele is initially at frequency 0.01 in teosinte and absent in maize. These parameter values were chosen to illustrate the dynamics of this model, and likely do not reflect the actual parameters in hybridizing $Z$. mays subspecies. Later, we show how varying parameter values shape the evolution of this form of reinforcement. See the Methods for additional detail.

\section{Sexual Conflict Leads to Transient Reinforcement}

When reinforcement evolves, it is almost always transient. Figure 2 shows the rise and fall of reinforcement, as well as allele and haplotype frequency dynamics across generations. Substantial reinforcement evolves when selection is strong, and reinforcement may even near completion (Phase 1, Fig. 2A). Reinforcement begins when the frequency of the female mating barrier, $F$, increases in teosinte (Fig. 2B) to prevent matings with foreign maladapted haplotypes from maize. This maintains the locally adaptive and pollen compatible haplotype in teosinte (Fig. 2C), building positive LD between alleles $A$ and $M$ (Fig. 2D).

The spread of the male compatibility allele, $M$, into maize (Phase 2 of Fig. 2) undermines the evolution of reinforcement. Initially, the female incompatibility allele, $F$, is rare and migrant $F$ alleles are disfavored in maize. This is because they preferentially mate with maladaptive migrant haplotypes from teosinte. As such, the mating advantage of the male compatibility allele
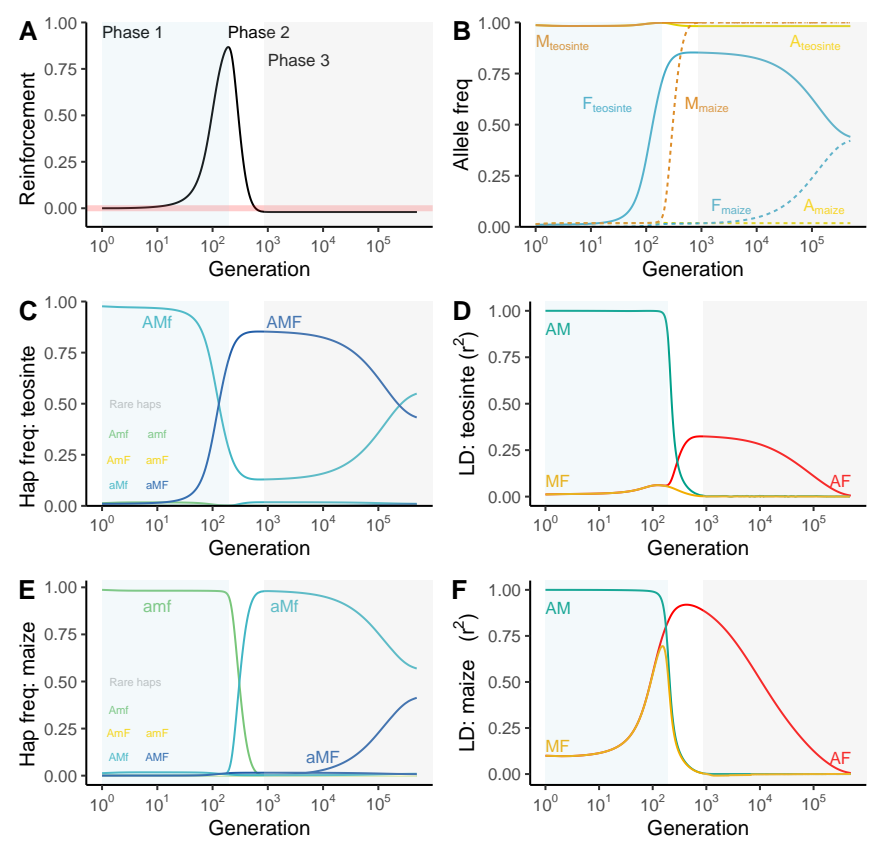

Figure 2 The rise and fall of reinforcement: We show the three phases of the transient evolution of reinforcement. First, a barrier preventing fertilization by $m$ pollen spreads in teosinte (light blue). Next, the compatible pollen allele, $M$, spreads in maize (white). After $M$ fixes, the barrier slowly disassociates from the teosinte background and eventually equilibriates in both populations (light grey). (A) Reinforcement is transient, building in Phase 1 and and breaking down in Phase 2. The pink line shows zero reinforcement. (B) Allele frequencies in both populations, with solid lines in teosinte and dashed lines in maize. The $F$ allele increases in teosinte, followed by fixation of $M$ and subsequent neutrality of $F$. (C) Haplotype frequencies in teosinte are largely due to underlying linkage disequilibrium (shown as $r^{2}$ in D). (E) Haplotype frequencies in maize, with linkage disequilibrium in maize shown in (F). This figure illustrates a single set of parameter values: Selection $-s_{\text {teo }}=s_{\text {maize }}=0.75$. Migration $\left(g_{\text {maize } \rightarrow \text { teo }}=g_{\text {teo } \rightarrow \text { maize }}=0.1\right)$. Recombination $-r_{\mathcal{A M}}=$ $r_{\mathcal{M F}}=0.0001$. Allele frequencies $-f_{M 0 \text {, teo }}=1, f_{M 0 \text {,maize }}=0$, $f_{F 0 \text {,teo }}=0.01, f_{F 0 \text {,maize }}=0$.

in maize is initially very small, due to the low frequency of the barrier allele, $F$. However, continued migration of $M$ into maize enables recombination of this allele onto the locally adaptive maize background (Fig. 2 E), decreasing LD between locally adaptive and pollen compatibility alleles (Fig. 2 F). This decrease in LD decreases selection against the stylar incompatibility allele in maize, allowing the modest mating advantage to facilitate the spread of the $M$ allele (Fig. 2B). As the $M$ allele rises in frequency and eventually fixes across the metapopulation, selection against the female barrier weakens until it is completely neutral. At this point it slowly equilibrates across populations by continued migration and recombination between local adaption and stylar incompatibility loci.

\section{Allele frequency change across the life cycle}

We now show how across the life cycle, migration, fertilization and selection drive changes in allele frequencies (Fig. 3). 

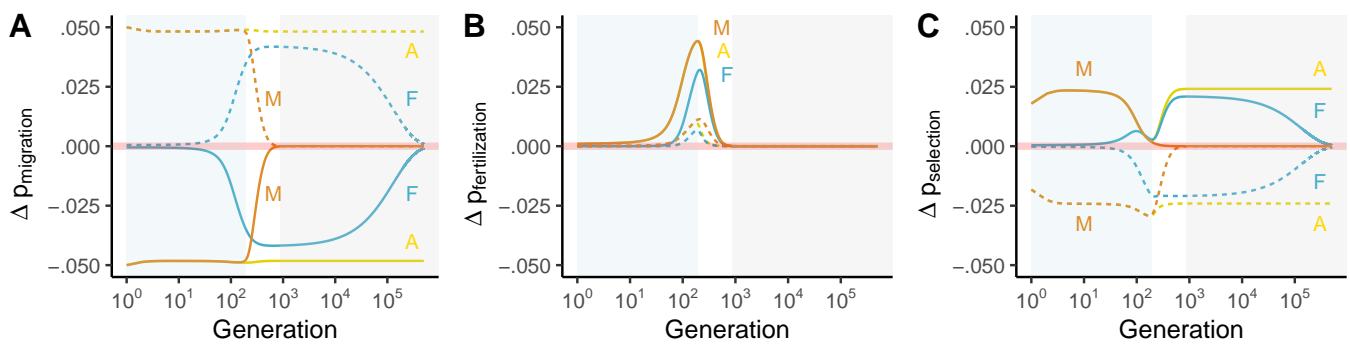

Subspecies

Allele

$A-F-M$

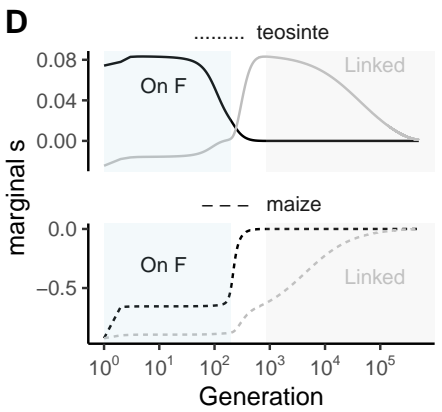

Figure 3 Allele frequency change across the life cycle: One locally adaptive allele with $s_{\text {teo }}=s_{\text {maize }}=0.75$, migration rates, $g_{\text {maize } \rightarrow \text { teo }}=g_{\text {teo } \rightarrow \text { maize }}=0.1$, and recombination rates $r_{\mathcal{A M}}=r_{\mathcal{M F}}=.0001$. Initially, populations are locally adapted $\left(f_{\mathcal{A} \text {,teo }}=\right.$ $\left.1-f_{\mathcal{A} \text {,maize }}=1\right)$, with $\mathrm{M}$ fixed in teo and absent in maize, and $\mathrm{F}$ at frequency 0.01 in teosinte and absent in maize. Background shading marks phase one (light blue), phase two (white), and phase three of transient reinforcement, as in Figure 2. Change in allele frequency during migration (A), fertilization (B), and selection (C), as described in main text, with the transparent thick pink line denoting no change in allele frequency during this life phase. (D) decomposes selection $F$ into it's effects on fitness by avoiding lowfitness hybrids, and its incidental linkage with selected haplotypes, as described in text and methods below.

Migration homogenizes allele frequencies between, and increases linkage disequilibrium within, populations. With allele (or haplotype) frequency $p_{\text {teo }}$ before pollen migration, allele frequency in the pollen pool after migration $p_{\text {teo }}^{\prime}$ is $p_{\text {teo }}^{\prime}=p_{\text {teo }}-$ $g_{\text {maize } \rightarrow \text { teo }}\left(p_{\text {teo }}-p_{\text {maize }}\right)$, where the prime symbol denotes frequency after migration. This homogenizing effect is clear in Figure $3 \mathrm{~A}$, as the frequency of all 'teosinte' alleles $(A, M$, and $F$, whose initial frequencies are 1,1 , and 0.01 in teosinte and 0 for all loci in maize; see Methods for details on robustness of these assumptions) decrease in teosinte (solid lines are all always less than zero) and increase in maize (dashed lines are always greater than zero).

The stylar barrier $F$ favors the male compatibility allele, $M$, via direct favoring of alleles overrepresented on $M$ haplotypes. The direct fertilization advantage to the $M$ allele is clear in Figure $3 \mathrm{~B}$ - in both populations the $M$ allele always increases more than any other allele. In addition to directly increasing the frequency of $M$ alleles, selection indirectly increases the frequency of alleles in LD with $M$. Because LD between all teosinte alleles is $>0$, a fertilization advantage indirectly favors both the $A$ and $F$ alleles in both maize and teosinte (at all loci in both populations, allele frequency change is always positive; Fig. 3B).

We now derive an analytical expectation for the mating advantage described in Fig. 3B). Assuming that the female postmating incompatibility allele, $F$ is dominant, the mating advantage of $M$ depends on the frequency of incompatible females, which are heterozygous or homozygous for $F$. We represent this frequency as $\left(1-p_{f f}\right)$, where $p_{f f}$, the frequency of styles homozygous for the $f$ allele, differs from $p_{f}^{2}$ due to assortative mating. As such, the increase in frequency of the $M$ allele in paternally derived haplotypes in the next generation (indicated by the prime symbol), and relative to pollen, equals

$$
p_{M^{\prime}} p_{m^{\prime}}\left(1-p_{f f}\right) \frac{c}{1-p_{m^{\prime}}}
$$

where $c$ is the intensity of discrimination against incompatible male-expressed alleles.

Allele frequency change by natural selection follows standard expectations under multiplicative fitness. Selection increases the frequency of the $A$ allele locally adapted to the teosinte environment in teosinte, and decreases its frequency in maize (Fig. 3C).
Given the many linkage associations generated by this system of migration, selection, and assortative mating (Fig. 2E,F), we seek to investigate the extent to which the increase in frequency of the female isolating barrier is attributable to selection for reinforcement (Hopkins et al. 2014), versus linked selection (see Methods). We find that the initial increase in frequency of the $F$ allele in teosinte is attributable to selection for reinforcement, but its persistence once $M$ has reached appreciable frequency in maize is attributable to linked selection (Fig. 3D). Both selection against this incompatibility and linked selection, acting on the $A$ locus, act to disfavor $F$ in maize. However, selection against $F$ weakens as $M$ rises in frequency in maize, and linked selection against $F$ weakens as recombination erodes LD between $F$ and $A$ (Fig. 3D). See Methods for details.

\section{Determinants of the strength and duration of reinforcement of postmating prezygotic isolation}

Above we have shown the basic dynamics of how sexual conflict results in the transient evolution of reinforcement. Here we show the parameters that modify the maximal amount (Fig. 4 A-D) and duration (Fig. 4 E-H) of reinforcement in the face of this conflict.

Reinforcement often requires strong selection: As selection on the locally adaptive allele intensifies, the maximum level and the duration of reinforcement both increase (Figures 4A and E, respectively). Under this set of parameters, the strength of selection on the locally adaptive locus must be exceptionally strong for any reinforcement to evolve - e.g. even with $s=0.1$ only very subtle reinforcement evolves for a very short time. However, we later show that other parameter choices - in particular, allowing for asymmetric migration - can weaken this requirement.

With symmetric migration and asymmetric selection, the strength of selection in maize generally has a greater effect on the extent and duration of reinforcement than does the strength of selection in teosinte (Fig. $4 \mathrm{~B}$ and F, respectively). This is because strong selection in maize initially keeps the frequency of the barrier allele low in maize, which minimizes the opportunity for the $M$ allele to recombine onto the locally adaptive background. This result holds over a broad range of symmetric migration rates (Supp. Fig. S1). 

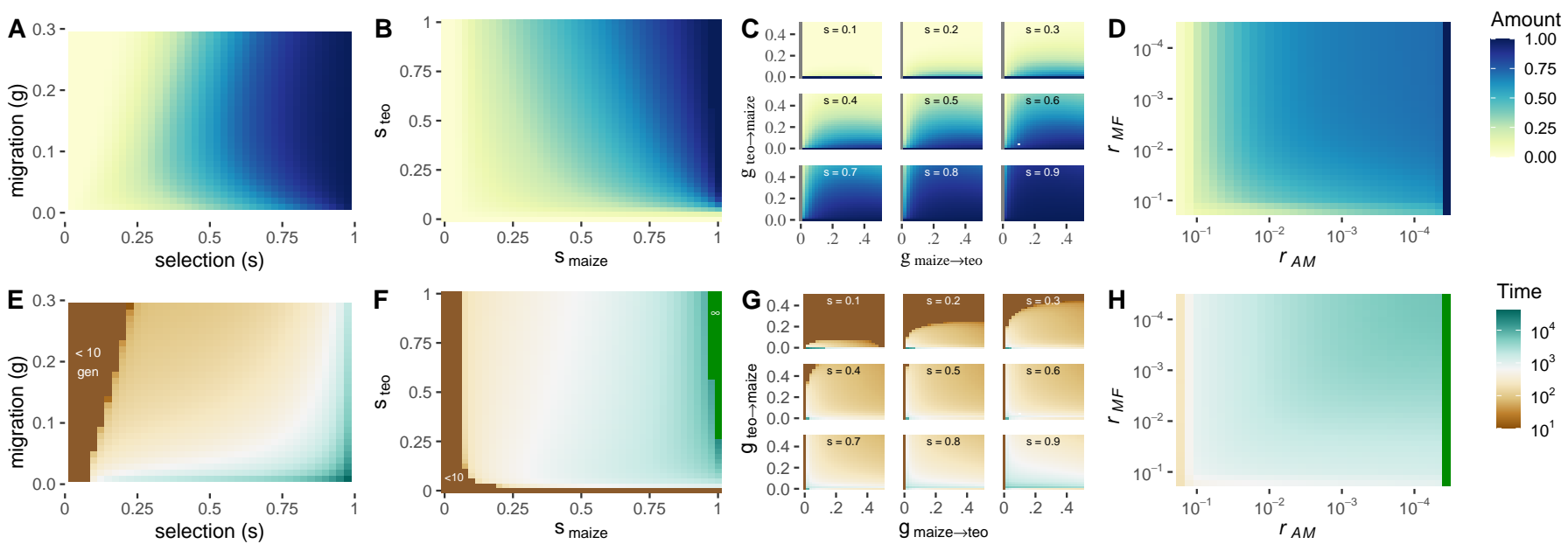

Figure 4 The maximum amount (A-D) and duration (E-H) of reinforcement: As a function of symmetric selection and migration rates $\left(\mathbf{A}\right.$ and $\mathbf{E}$ with $\left.r_{\mathcal{A M}}=r_{\mathcal{M F}}=10^{-4}\right)$, different selection coefficients in maize and teosinte $\left(\mathbf{B}\right.$ and $\mathbf{F}$, with $g_{\text {maize } \rightarrow \text { teo }}=$ $g_{\text {teo } \rightarrow \text { maize }}=0.03$ and $\left.r_{\mathcal{A M}}=r_{\mathcal{M F}}=10^{-4}\right)$, asymmetric migration rates, across numerous selection coefficients $(\mathbf{C}$ and $\mathbf{G}$, with $\left.r_{\mathcal{A M}}=r_{\mathcal{M F}}=10^{-4}\right)$, and recombination rates $\left(\mathbf{D}\right.$ and $\mathbf{H}$, with a symmetric selection coefficient of 0.8 and $g_{\text {maize } \rightarrow \text { teo }}=$ $g_{\text {teo } \rightarrow \text { maize }}=0.03$ ). We note that the x-axis in figures $\mathbf{D}$ and $\mathbf{H}$ moves from loose linkage on the left to tight linkage on the right. Complete or non-transient reinforcement is visible on the far right of figures $\mathbf{F}$ and $\mathbf{H}$, indicated by the darkest green time duration, and the $\infty$ symbol in $\mathbf{F}$. The amount of reinforcement is quantified as $\left.\left(p_{[z \text {,gen }=\mathbf{x}]}-p_{[z \text {,gen=0 }}\right]\right) / p_{[z \text {,gen=0] }}, p_{z}$ equals the probability of being fertilized by non-migrant pollen scaled by the frequency of non-migrant pollen grains, and gen refers to time in generations.

Reinforcement is maximized with intermediate migration rates: Regardless of selection coefficient, we find that intermediate symmetric migration rates always maximize the extent of reinforcement (Fig. 4A), while the duration of reinforcement decreases as the migration rate increases (Fig. 4E).

Examining the effect of asymmetric migration on the extent of reinforcement highlights the role of migration in resolving conflict. With low migration, increasing migration from maize to teosinte results in a higher intensity of reinforcement. By contrast, increasing migration from teosinte to maize more strongly reduces the maximum level of reinforcement, especially at higher migration rates (Fig. 4C).

More reinforcement evolves with tight linkage between the male compatibility allele and a single locally adaptive allele: With tight linkage between the locally adaptive locus $\mathcal{A}$ and the compatibility allele $\mathcal{M}\left(r_{\mathcal{A M}}=r_{\mathcal{A F}}=10^{-4}\right)$, substantial reinforcement can evolve and last for some time. However, the strength and duration of reinforcement drops, initially modestly, and then quite precipitously, as loci become more genetically distant, with nearly no reinforcement when $\mathcal{A}$ and $\mathcal{M}$ are unlinked (Fig. $4 \mathrm{D}, \mathrm{H})$. The selection coefficient modulates this effect of recombination (Fig. S2) - when selection is very strong (e.g. s > 0.6) some reinforcement can evolve, even when $\mathcal{A}$ and $\mathcal{M}$ are separated by up to a centiMorgan (i.e. $r_{A M}=0.01$ ).

This result suggests that the recombination rate between the locally adaptive locus and the male compatibility allele, $r_{\mathcal{A M}}$, plays a critical role in mediating the sexual conflict over reinforcement. When $r_{\mathcal{A M}}$ is high, meaning $\mathcal{A}$ and $\mathcal{M}$ are loosely linked, the male benefit to overcoming the female barrier is increased. This is because the haplotypes formed in later generation offspring are highly likely to be locally adapted, as the pollen compatibility allele $M$ can escape from a locally maladapted genomic background.

Linkage between female barrier and male (in)compatibility alleles does not strongly impact the amount or duration of reinforcement:
Linkage between the male and female (in)compatibility alleles, $r_{\mathcal{M F}}$, has a more subtle effect on the extent and duration of reinforcement than does $r_{\mathcal{A M}}$. Across most selection coefficients and most values of $r_{\mathcal{A M}}$, the rate of recombination between $\mathcal{F}$ and $\mathcal{M}$ only modestly impacts the amount and duration of reinforcement (Supp. Fig. S2).

The presence of multiple unlinked adaptive loci increases reinforcement: Our results so far suggest that the (transient) evolution of postmating prezygotic isolation requires tight linkage between differentially selected loci and loci underlying this incompatibility. However, the genetic architecture of local adaptation is often polygenic (Barghi et al. 2020), and this may even be the case in some maize traits Wallace et al. (2014). Thus tight linkage between locally adaptive alleles and gametic incompatibilities may be biologically unrealistic.

We therefore investigate if weaker selection at more unlinked loci can allow reinforcement to transiently evolve. We show in Figure 5 that reinforcement can evolve for some time when numerous unlinked alleles experience divergent selection in the two populations. While the selection coefficients displayed are still quite large, our results suggest that weaker selection at many loci could likely result in the transient evolution of reinforcement.

\section{Discussion}

For decades, researchers have presented theoretical and empirical challenges to the process of reinforcement, starting with a foundational paper from Felsenstein (1981), who identified recombination as a critical challenge to reinforcement. Since then, a large body of theory has aimed to identify the circumstances that permit reinforcement to evolve, along with those that hinder it (reviewed in Kirkpatrick and Ravigné 2002). Despite its role in hampering speciation, sexual conflict over hybridization has received little attention in the literature, usually as a brief 

available under aCC-BY-NC-ND 4.0 International license.
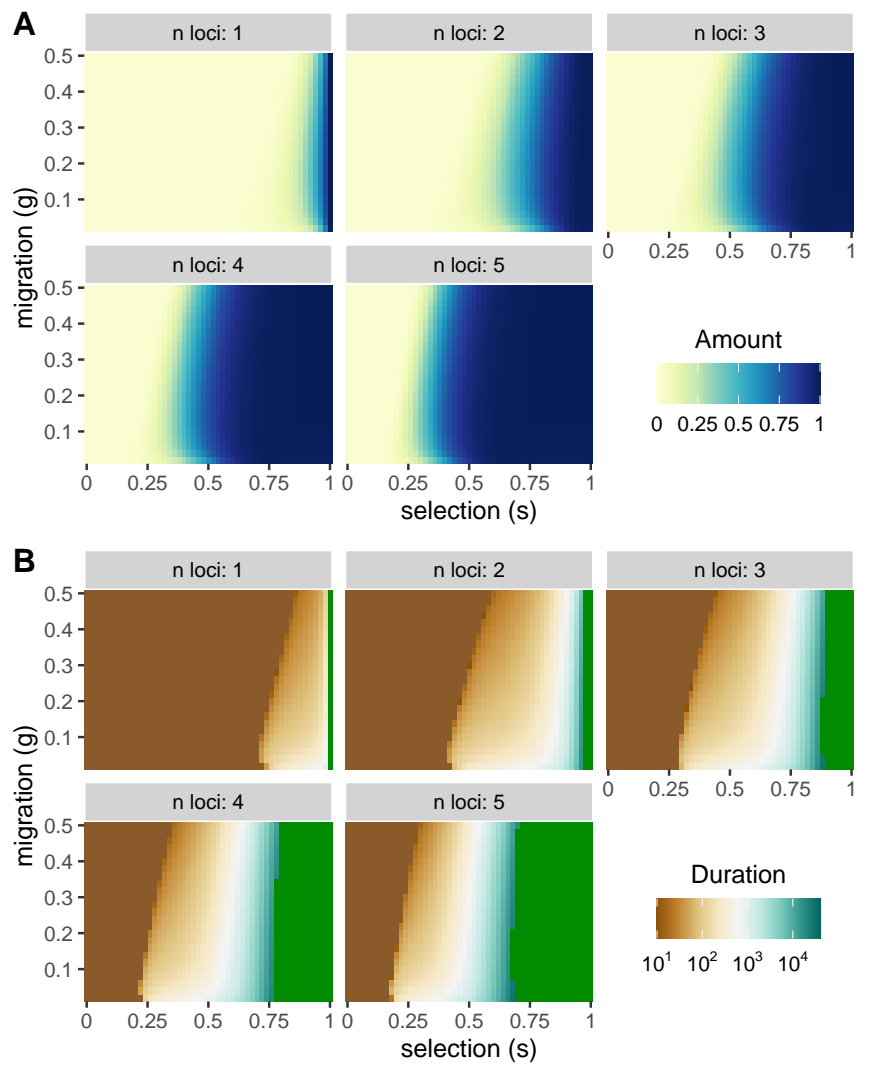

Figure 5 Oligogenic ecological selection: The maximum strength, $A$ and duration, $B$, of reinforcement with ecological selection at $\mathrm{n}$ loci, where all ecologically selected loci are unlinked to one another and to the gametophytic factor locus. The selection coefficient against a maladaptive allele, $s$, is multiplicative within and among loci. Migration rate, $g$, is symmetric and recombination rate $r_{\mathcal{A M}}=10^{-4}$.

aside in papers concerning the role of sexual conflict in speciation more broadly (Parker and Partridge 1998; Gavrilets and Hayashi 2005; Gavrilets 2014). We identify the transient dynamics generated by sexual conflict over reinforcement and the evolutionary traces it leaves behind - namely the adaptive spread of a female barriers in one species/population and the adaptive introgression of male compatibility alleles into the other.

These results provide a rich set of predictions and interpretations of empirical patterns that were missed by previous game theoretic (Parker and Partridge 1998; Gavrilets and Hayashi 2005) and verbal (Coyne and Orr 2004) models. We show that sexual conflict favors male gametic traits that overcome heterospecific female barriers. This conflict arises when female gametes are selected to avoid hybridization, while male gametes are selected to mate as often as possible. The breakdown of reproductive isolation is marked by the rapid adaptive introgression of the male compatibility factor into maize, following recombination onto the locally adaptive haplotype. Backmigration of this allele into the teosinte population hastens its fixation across populations. The final step in this model is slow homogenization of the female barrier allele across species, as the male compatibility allele fixes in both populations, rendering the isolating barrier ineffective. Ultimately, we show that barriers acting at different stages of hybridization can affect how reinforcement proceeds. Below we discuss the relationship of our results to previous theory, implications for the process of reinforcement, and empirical implications for hybridizing Zea mays subspecies and other taxa.

\section{Comparison to previous models}

Mechanisms of assortative mating: Previous models of reinforcement treated the sexes interchangeably (Felsenstein 1981), or assumed assortative mating under female control (Lande 1981; Servedio and Kirkpatrick 1997; Kelly and Noor 1996), either by "matching" or "preference/trait" mechanisms of assortative mating (Kopp et al. 2018). Both matching and preference/trait models implicitly induce a trade-off between interspecific and intraspecific mating - a male with a trait favored by heterospecific females will have limited mating success with conspecific females. One major distinguishing feature of our model is the mechanism of assortative mating, a lock and key model of reproductive isolation that underlies well-characterized cases of postmating prezygotic isolation in e.g. Zea mays subspecies (Kermicle 2006) as well as broadcast spawners (e.g. Lessios 2007). In this model, introgression of this male compatibility allele is facilitated by the fact that it does not prevent intraspecific mating - that is, there is no evolutionary trade-off between intra- and interspecific mating. As such, while lock and key type mating interactions can result in the transient evolution of reinforcement, this transience does not arise in matching or preference/trait models.

Sexual conflict and speciation: The role of sexual conflict in removing species boundaries runs counter to the conventional role it is thought to play in speciation (Parker and Partridge 1998; Van Doorn et al. 2001; Gavrilets and Waxman 2002; Rice 1996). Previous theory (Gavrilets and Waxman 2002) and experiments (Palopoli et al. 2015), as well as natural patterns of reproductive isolation (Brandvain and Haig 2005; Ting et al. 2014) and diversification rates (Arnqvist et al. 2000) suggested that sexually antagonistic coevolution can lead to coevolutionary arms races in each incipient species, pleiotropically resulting in behavioral or mechanical isolation. In this manner, intraspecific sexual conflict was thought to be an "Engine of Speciation" (Rice et al. 2005). By contrast, we show that the interspecific conflict between the sexes over fertilization hampers speciation by preventing the evolution of reinforcement. More broadly, our results align with studies suggesting that incompatibilities (Bank et al. 2012), especially those with a transmission advantage (Meiklejohn et al. 2018), can adaptively introgress across species boundaries.

Comparison to the "counter-intuitive role of Fisherian selection": Servedio and Bürger (2014) found that Fisherian sexual selection can undermine the evolution of assortative mating, and specifically that introgression of female preference across species can favor heterospecific male traits by sexual selection even when such traits are disfavored by natural selection. Their model incorporates a mating advantage to maladaptive male traits, which undermines the evolution of reinforcement. In contrast, our model shows that the benefit of siring low fitness hybrids, as opposed to being attractive to rare conspecifics, can also undermine the evolution of reinforcement. Furthermore, unlike Servedio and Bürger (2014), the stylar barrier allele in our model is at low frequency for a substantial period of time in maize, as it is disfavored while the locally adaptive trait is in linkage disequilibrium with the male compatibility allele. 

available under aCC-BY-NC-ND 4.0 International license.

\section{Implications for the process of reinforcement}

The importance of opportunity cost in the reinforcement of premating vs. postmating prezygotic isolation: Most models of reinforcement of premating isolation include a suite of similar assumptions and traits. They incorporate either matching or preference/trait models (Kopp et al. 2018), focus on premating isolation, and include a strict tradeoff in inter- versus intraspecific matings, hiding differences in the costs to hybridization experienced by males and females (but see Spencer et al. 1986, as a notable exception).

In general, premating barriers will incur more similar opportunity costs to males and females, thus minimizing sexual conflict, while opportunity costs might differ more broadly for postmating isolation. Similarly, mechanisms underlying postmating prezygotic isolation may themselves include trade-offs, allowing for evolutionary stability of reinforcement. That is, certain physical and/or biochemical properties of postmating prezygotic interactions may minimize the opportunity for interspecific sexual conflict by enforcing a trade-off between overcoming a heterospecific barrier and successfully fertilizing conspecifics. Such a case is particularly likely under competitive fertilization - where a gamete could trade off interspecific competitive success for intraspecific competitive success. As Howard (1993) argued, and Lorch and Servedio (2007) showed, a preference for conspecific sperm can stably evolve to minimize interspecific fertilization. Thus we expect the stable reinforcement of postmating prezygotic isolation by conspecific sperm precedence, as observed between sympatric populations of D. pseudoobscura and D. persimilis (Castillo and Moyle 2019). Finally, we note that even non-competitive fertilization can induce a trade-off between inter- and intraspecific crossing success. For example, if pollen must grow an optimal distance to fertilize an ovule (as observed in interspecific Nicotiana crosses Lee et al. 2008), it will not be able to simultaneously succeed on both inter- and intraspecific styles.

\section{Limitations and caveats:}

Our model is inspired by the empirical genetics of postmating prezygotic isolation observed between Zea mays subspecies. Still we warn that we have simplified this system for analysis, and that parameter values and modelling choices in this model are not carefully calibrated to this, $r$ any her system. Most notably, we assumed only two populations and a simple multiplicative fitness function across a small number of divergently selected loci. In general, our model revealed that strong ecological selection was required for the initial reinforcement of postmating prezygotic isolation. However, this requirement became less stringent as we included more loci.

The appropriate model for the genetic architecture of adaptive differentiation, and the linkage between locally adaptive alleles and gametic isolation, is biologically variable, and unknown in this system. tga1 and su1, two 'domestication' loci likely experiencing strong divergent selection in maize and teosinte, are within 10 centiMorgans of one well-characterized gametophytic factor, suggesting that strong selection on locally adaptive loci is plausible in this system Wang et al. (2005); Whitt et al. (2002). However, Z. mays' other gametophytic factors are not closely linked to loci known to experience strong divergent selection. Outside of this species pair, adaptive differentiation may be under the control of few loci (Selby and Willis 2018; Lowry and Willis 2010) or many (Yeaman 2013).

As such, future work could focus on evaluating how this system scales with a large number of adaptive loci sprinkled across a realistic genome and/or population structure. Additionally, rather than looking at simple extrinsic isolation, future work could evaluate the extent to which our results generalize to Dobzhansky-Muller Incompatibilities, or other genetic architectures of isolation.

Additionally, we limited our analysis to one postmating prezygotic barrier, with a simple mode of gene action. But at least three gametophytic factor loci exist in Z. mays subspecies, and across these, alternative haplotypes are found in different frequencies in different populations (e.g. Kermicle 2006; Kermicle et al. 2006; Kermicle and Evans 2010). Why several such loci exist, and how they interact, remain open questions.

\section{Empirical implications, novel predictions, and interpretation of current observations}

Our model shows that the common biological phenomenon of sexual conflict (see Arnqvist and Rowe 2005, for examples), wherein male and female interests are misaligned, may be sufficient to erode reinforcement via postmating prezygotic isolation. These results highlight an under-appreciated challenge to the reinforcement of postmating prezygotic barriers. Convincing cases of the reinforcement of postmating prezygotic isolation provide a strong opportunity to further test our theory. Specifically, our results predict that such cases are either unstable (and therefore recent) or will should involve: (1) unidirectional gene flow, (2) a trade-off between male success in overcoming inter- and intraspecific postmating barriers, as is found in preference/trait mechanisms, and/or (3) exceptionally strong postzygotic isolation, such that gene flow is vanishingly rare.

Interpretation of empirical patterns: The precariousness of reinforcing postmating prezygotic isolation shown in our model is consistent with the meta-analyses showing that postmating prezygotic isolation does not differ between sympatric and allopatric species pairs across three angiosperm genera (Moyle et al. 2004) or in Drosophila (Turissini and Matute 2017). Our results are also consistent with the growing consensus that, contrary to initial claims (e.g. Geyer and Palumbi 2003), reinforcement may not drive the evolution of sperm-egg interactions underlying reproductive barriers in broadcast spawners (see discussion in Vacquier and Swanson 2011). This highlights the difficulties inherent to the stable reinforcement of gametic isolation.

While the rarity of reinforcement of postmating prezygotic isolation is consistent with our theory, the few documented cases in which postmating prezygotic barriers are reinforced allow for better evaluation of our model. Kay (Kay and Schemske 2008; Yost and Kay 2009) has provided strong evidence consistent with the reinforcement of postmating prezygotic isolation between the wild ginger, C. scaber and sympatric C. pulverulentus. Consistent with expectations of our model, gene flow in this case is unidirectional (Kay 2006).

Poikela et al. (2019) found elevated postmating prezygotic isolation only in D. flavomontana populations recently sympatric with $D$. montana, and premating isolation is only reinforced in populations which have been sympatric for longer periods. Together, these observations are consistent with our prediction that reinforcement of postmating prezygotic isolation will be transient. The reinforcement of postmating prezygotic isolation between $D$. yakuba and $D$. santomea is most difficult to reconcile with our model, as they have a stable hybrid zone, no evidence 
of conspecific sperm precedence, and bidirectional hybridization (Matute 2010)). However, Turissini and Matute (2017) observed only short (i.e. very old) blocks of introgression in this species pair, suggesting minimal ongoing gene flow, and exceptionally strong postzygotic isolation in nature. In sum, existing cases suggesting the reinforcement of postmating prezygotic isolation are largely consistent with our theoretical predictions.

Predictions for empirical systems: Domesticated maize landraces (Z. m. ssp. mays) and teosinte (Z. m. ssp. mexicana) grow in close proximity in the Mexican highlands, where Z. $m$. ssp. mexicana grows as an agricultural weed. As described in the introduction, the biology of this system is favorable to the evolution of reinforcement - as gene flow occurs between subspecies and hybrids have reduced but non-zero fitness in either parental environment (Stitzer and Ross-Ibarra 2018). Stylar incompatibilities are common in Z. $m$. ssp. mexicana sympatric with maize, but "[ $t]$ he unexpected presence of the [male compatibility] allele in sympatric landrace maize appear[ed]... to negate any effect of [the] crossing barrier" puzzling researchers (Kermicle et al. 2006). Our model explains this observation as the adaptive introgression of teosinte pollen compatibility alleles into maize. We suggest that in most sympatric populations, at most gametophytic factors, the stylar mating barrier has already rapidly swept through Z. $m$. ssp. mexicana (Phase 1 in Figure 2), and the pollen incompatibility has adaptively introgressed into sympatric highland maize landraces (Phase 2 in Figure 2. In this case, our expectation from empirical observations suggests that these populations are in the slow final phase of our model, in which allele frequencies at the stylar barrier slowly equilibrate.

This interpretation makes specific testable predictions concerning the ancestry and patterns of selection near gametophytic factors in sympatric maize and teosinte populations in the Mexican highlands. For example, we predict the adaptive introgression of male compatibility alleles from teosinte into maize, and selection against the teosinte stylar barrier in a maize background.

\section{Materials and Methods}

Gametophytic factors: Our notation throughout differs from the existing literature on gametophytic factors. In this literature, each of the three known gametophytic factors (Ga1, Ga2, and Tcb1) is represented as a single locus with three haplotypes (e.g. Moran Lauter et al. 2017). The "strong"' haplotype, indicated by $s$ or $S$, contains both functional alleles $F$ and $M$. The haplotype with only the pollen-expressed compatibility allele is referred to as $m$, and is equivalent to the $M f$ genotype in the present study. The non-functional allele is referred to in the literature in lowercase, and is equivalent to the $m f$ genotype in our study. The $m F$ genotype is not known in the wild.

Additional Assumptions: We forward iterated a deterministic population genetic model. We assumed that the stylar incompatibility allele was initially rare in teosinte $\left(p_{\text {Fteo,gen }}=0.01\right.$ and absent in maize, that both populations were initially well adapted to their environments (with appropriate local adaptation allele fixed at all $\mathcal{A}$ loci), and that the pollen compatibility allele, $M$, was initially fixed in teosinte and absent in maize. We were initially concerned that our results could be sensitive to the initial frequency of the pollen compatibility allele, however, preliminary explorations found nearly no effect of this parameter, presumably because it rapidly sweeps through teosinte (Fig.
S3). For all results in the main text, we assumed that discrimination was complete, i.e. that styles with barrier genotypes could not be fertilized by incompatible pollen (i.e. $c=1$ ). Relaxing this assumption results in expected quantitative differences in results (e.g. reinforcement evolves to lower levels), but does not change our qualitative conclusions (Fig. S4).

Quantifying reinforcement and its duration: We summarized much of our results by quantifying the maximum extent and duration of reinforcement. We quantified the amount of reinforcement at generation $x$ as $\left.\left(p_{[z \text {,gen }=\mathrm{x}]}-p_{[z \text {,gen }=0}\right]\right) / p_{[z \text {,gen }=0]}$, $p_{z}$ equals the probability of being fertilized by non-migrant pollen scaled by the frequency of non-migrant pollen grains. We quantified the duration of reinforcement as the number of generations for which the amount of reinforcement was greater than 0.05 .

Computer Code: All code is written in $\mathrm{R}$
$(\mathrm{R}$ Core Team 2020) and is available at https://github.com/carushworth/gftheory1. We generated figures with the ggplot2 (Wickham 2016) and cowplot (Wilke 2020) packages, and used the dplyr (Wickham et al. 2020) package to process numeric results.

Partitioning selection: To quantify the strength of selection for (or against) female incompatibility, we calculate the difference in mean fitness of zygotes with maternal derived $F$ and $f$ alleles for each of each two-locus $\mathcal{A M}$ maternal haplotype, and weight this difference by the frequency of this two-locus haplotype in ovules before selection $\left(\Sigma \Sigma f_{\mathcal{A}_{\rangle} \mathcal{M}_{1} \ldots \ldots .} \times\left(\bar{w}_{A_{i} M_{j} F / \ldots}-\bar{w}_{A_{i} M_{j} f / \ldots}\right)\right.$, with phased maternal/paternal genotypes). In so doing, we control for all aspects of maternal genotype, except for its impact on patterns of mating.

To quantify the strength of linked selection on female incompatibility, we calculate the difference in mean fitness of zygotes with maternal derived $F$ and $f$ alleles for each of each three-locus $\mathcal{A M F}$ paternal haplotype, and weight this difference by the frequency of this three-locus haplotype in ovules

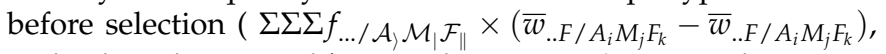
with phased maternal/paternal genotypes). In so doing, we control for the direct impact of the $F$ allele on assortative mating, and only measure the impact of its genetic association.

\section{Acknowledgments}

This work was funded by NSF award \#1753632 to J Ross-Ibarra, MMS Evans, and Y Brandvain. We thank Robin Hopkins and Maria Servedio, whose comments greatly improved the manuscript.

\section{References}

Arnqvist, G., M. Edvardsson, U. Friberg, and T. Nilsson, 2000 Sexual conflict promotes speciation in insects. Proceedings of the National Academy of Sciences 97: 10460-10464.

Arnqvist, G. and L. Rowe, 2005 Sexual conflict. Princeton University Press.

Bank, C., R. Bürger, and J. Hermisson, 2012 The limits to parapatric speciation: Dobzhansky-muller incompatibilities in a continent-island model. Genetics 191: 845-63.

Barghi, N., J. Hermisson, and C. Schlötterer, 2020 Polygenic adaptation: a unifying framework to understand positive selection. Nature Reviews Genetics pp. 1-13. 
Brandvain, Y. and D. Haig, 2005 Divergent mating systems and parental conflict as a barrier to hybridization in flowering plants. Am Nat 166: 330-8.

Castillo, D. M. and L. C. Moyle, 2019 Conspecific sperm precedence is reinforced, but postcopulatory sexual selection weakened, in sympatric populations of drosophila. Proc Biol Sci 286: 20182535.

Clifton, K. E., 1997 Mass spawning by green algae on coral reefs. Science 275: 1116-1118.

Coyne, J. A., 1974 The evolutionary origin of hybrid inviability. Evolution 28: 505-506.

Coyne, J. A. and A. Orr, 2004 Speciation. Sinauer.

Coyne, J. A. and H. A. Orr, 1989 Patterns of speciation in Drosophila. Evolution 43: 362-381.

Coyne, J. A. and H. A. Orr, 1997 "patterns of speciation in Drosophila" revisited. Evolution 51: 295-303.

Dobzhansky, T., 1937 Genetics and the Origin of Species. Columbia University Press.

Ellstrand, N. C., L. C. Garner, S. Hegde, R. Guadagnuolo, and L. Blancas, 2007 Spontaneous Hybridization between Maize and Teosinte. Journal of Heredity 98: 183-187.

Felsenstein, J., 1981 Skepticism towards santa rosalia, or why are there so few kinds of animals? Evolution 35: 124-138.

Gavrilets, S., 2014 Is sexual conflict an "engine of speciation"? Cold Spring Harb Perspect Biol 6: a017723.

Gavrilets, S. and T. Hayashi, 2005 Speciation and sexual conflict. Evolutionary Ecology 19: 167-198.

Gavrilets, S. and D. Waxman, 2002 Sympatric speciation by sexual conflict. Proc Natl Acad Sci U S A 99: 10533-8.

Geyer, L. and S. Palumbi, 2003 Reproductive character displacement and the genetics of gamete recognition in tropical sea urchins. Evolution 57: 1049-60.

Hopkins, R., 2013 Reinforcement in plants. New Phytol 197: 1095-103.

Hopkins, R., R. Guerrero, M. Rausher, and M. Kirkpatrick", 2014 Strong reinforcing selection in a texas wildflower. Current Biology 24: 1995 - 1999.

Howard, D. J., 1993 Reinforcement: origin, dynamics, and fate of an evolutionary hypothesis. In Hybrid zones and the evolutionary process, edited by R. G. Harrison, pp. 46-69, Oxfprd University Press, Oxford.

Johnson, N. A., 2008 Direct selection for reproductive isolation: the wallace effect and reinforcement. In Natural Selection and Beyond: The Intellectual Legacy of Alfred Russel Wallace, edited by C. H. Smith and G. Beccaloni, Oxford University Press.

Johnson, N. A. and M. J. Wade, 1995 Conditions for soft selection favoring the evolution of hybrid inviability. Journal of Theoretical Biology 176: 493 - 499.

Kay, K. M., 2006 Reproductive isolation between two closely related hummingbird-pollinated neotropical gingers. Evolution 60: 538-52.

Kay, K. M. and D. W. Schemske, 2008 Natural selection reinforces speciation in a radiation of neotropical rainforest plants. Evolution 62: 2628-42.

Kelly, J. K. and M. A. Noor, 1996 Speciation by reinforcement: a model derived from studies of drosophila. Genetics 143: 1485-97.

Kermicle, J., S. Taba, and M. Evans, 2006 The gametophyte-1 locus and reproductive isolation among zea mays subspecies. Maydica 51: 219-225.

Kermicle, J. L., 2006 A selfish gene governing pollen-pistil compatibility confers reproductive isolation between maize rela- tives. Genetics 172: 499-506.

Kermicle, J. L. and M. M. S. Evans, 2010 The zea mays sexual compatibility gene ga2: Naturally occurring alleles, their distribution, and role in reproductive isolation. Journal of Heredity 101: 737-749.

Kirkpatrick, M., 2001 Reinforcement during ecological speciation. Proc Biol Sci 268: 1259-63.

Kirkpatrick, M. and V. Ravigné, 2002 Speciation by natural and sexual selection: models and experiments. Am Nat 159 Suppl 3: S22-35.

Kopp, M., M. R. Servedio, T. C. Mendelson, R. J. Safran, R. L. Rodríguez, et al., 2018 Mechanisms of assortative mating in speciation with gene flow: Connecting theory and empirical research. Am Nat 191: 1-20.

Lande, R., 1981 Models of speciation by sexual selection on polygenic traits. Proceedings of the National Academy of Sciences 78: 3721-3725.

Lee, C. B., L. E. Page, B. A. McClure, and T. P. Holtsford, 2008 Post-pollination hybridization barriers in Nicotiana section Alatae. Sexual Plant Reproduction 21: 183.

Lessios, H., 2007 Reproductive isolation between species of sea urchins. Bulletin of Marine Science 81: 191-208.

Liou, L. W. and T. D. Price, 1994 Speciation by reinforcement of premating isolation. Evolution 48: 1451-1459.

Lorch, P. D. and M. R. Servedio, 2007 The evolution of conspecific gamete precedence and its effect on reinforcement. J Evol Biol 20: 937-49.

Lowry, D. B. and J. H. Willis, 2010 A widespread chromosomal inversion polymorphism contributes to a major life-history transition, local adaptation, and reproductive isolation. PLOS Biology 8: 1-14.

Lu, Y., S. A. Hokin, J. L. Kermicle, T. Hartwig, and M. M. S. Evans, 2019 A pistil-expressed pectin methylesterase confers cross-incompatibility between strains of Zea mays. Nature Communications 10: 2304.

Lu, Y., J. L. Kermicle, and M. M. S. Evans, 2014 Genetic and cellular analysis of cross-incompatibility in zea mays. Plant Reprod 27: 19-29.

Lu, Y., A. N. Moran Lauter, S. Makkena, M. P. Scott, and M. M. S. Evans, 2020 Insights into the molecular control of cross-incompatibility in Zea mays. Plant Reproduction .

Mangelsdorf, P. C. and D. F. Jones, 1926 The expression of mendelian factors in the gametophyte of maize. Genetics 11: 423-55.

Matute, D. R., 2010 Reinforcement of gametic isolation in Drosophila. PLoS Biol 8: e1000341.

Meiklejohn, C. D., E. L. Landeen, K. E. Gordon, T. Rzatkiewicz, S. B. Kingan, et al., 2018 Gene flow mediates the role of sex chromosome meiotic drive during complex speciation. Elife 7.

Metz, E. C., R. E. Kane, H. Yanagimachi, and S. R. Palumbi, 1994 Fertilization between closely related sea urchins is blocked by incompatibilities during sperm-egg attachment and early stages of fusion. Biol Bull 187: 23-34.

Moran Lauter, A. N., M. G. Muszynski, R. D. Huffman, and M. P. Scott, 2017 A pectin methylesterase zmpme3 is expressed in gametophyte factor1-s (ga1-s) silks and maps to that locus in maize (Zea mays 1.). Frontiers in Plant Science 8: 1926.

Moyle, L. C., M. S. Olson, and P. Tiffin, 2004 Patterns of reproductive isolation in three angiosperm genera. Evolution 58: 1195-208.

Palopoli, M. F., C. Peden, C. Woo, K. Akiha, M. Ary, et al., 2015 
Natural and experimental evolution of sexual conflict within Caenorhabditis nematodes. BMC Evolutionary Biology 15: 93.

Parker, G. A. and L. Partridge, 1998 Sexual conflict and speciation. Philos Trans R Soc Lond B Biol Sci 353: 261-74.

Paterniani, E., 1969 Selection for reproductive isolation between two populations of maize, Zea mays 1. Evolution 23: 534-547.

Poikela, N., J. Kinnunen, M. Wurdack, H. Kauranen, T. Schmitt, et al., 2019 Strength of sexual and postmating prezygotic barriers varies between sympatric populations with different histories and species abundances. Evolution 73: 1182-1199.

R Core Team, 2020 R: A Language and Environment for Statistical Computing. R Foundation for Statistical Computing, Vienna, Austria.

Rice, W. R., 1996 Sexually antagonistic male adaptation triggered by experimental arrest of female evolution. Nature 381: 232-4.

Rice, W. R., J. E. Linder, U. Friberg, T. A. Lew, E. H. Morrow, et al., 2005 Inter-locus antagonistic coevolution as an engine of speciation: assessment with hemiclonal analysis. Proc Natl Acad Sci U S A 102 Suppl 1: 6527-34.

Selby, J. P. and J. H. Willis, 2018 Major qtl controls adaptation to serpentine soils in mimulus guttatus. Molecular Ecology 27: 5073-5087.

Servedio, M. R. and R. Bürger, 2014 The counterintuitive role of sexual selection in species maintenance and speciation. Proc Natl Acad Sci U S A 111: 8113-8.

Servedio, M. R. and M. Kirkpatrick, 1997 The effects of gene flow on reinforcement. Evolution 51: 1764-1772.

Servedio, M. R. and M. A. Noor, 2003 The role of reinforcement in speciation: Theory and data. Annual Review of Ecology, Evolution, and Systematics 34: 339-364.

Slaughter, C., M. A. McCartney, and P. O. Yund, 2008 Comparison of gamete compatibility between two blue mussel species in sympatry and in allopatry. Biol Bull 214: 57-66.

Spencer, H. G., B. H. McArdle, and D. M. Lambert, 1986 A theoretical investigation of speciation by reinforcement. The American Naturalist 128: 241-262.

Stitzer, M. C. and J. Ross-Ibarra, 2018 Maize domestication and gene interaction. New Phytologist 220: 395-408.

Swanson, W. J. and V. D. Vacquier, 1998 Concerted evolution in an egg receptor for a rapidly evolving abalone sperm protein. Science 281: 710-2.

Ting, J. J., G. C. Woodruff, G. Leung, N.-R. Shin, A. D. Cutter, et al., 2014 Intense sperm-mediated sexual conflict promotes reproductive isolation in caenorhabditis nematodes. PLOS Biology 12: 1-14.

Turissini, D. A. and D. R. Matute, 2017 Fine scale mapping of genomic introgressions within the drosophila yakuba clade. PLOS Genetics 13: 1-40.

Turissini, D. A., J. A. McGirr, S. S. Patel, J. R. David, and D. R. Matute, 2017 The Rate of Evolution of Postmating-Prezygotic Reproductive Isolation in Drosophila. Molecular Biology and Evolution 35: 312-334.

Turner, E., D. J. Jacobson, and J. W. Taylor, 2010 Reinforced postmating reproductive isolation barriers in neurospora, an ascomycete microfungus. Journal of Evolutionary Biology 23: 1642-1656.

Vacquier, V. D. and W. J. Swanson, 2011 Selection in the rapid evolution of gamete recognition proteins in marine invertebrates. Cold Spring Harbor Perspectives in Biology 3: a002931.
Van Doorn, G. S., P. C. Luttikhuizen, and F. J. Weissing, 2001 Sexual selection at the protein level drives the extraordinary divergence of sex-related genes during sympatric speciation. Proc Biol Sci 268: 2155-61.

Wallace, A. R., 1889 Darwinism. Macmillan.

Wallace, J., S. Larsson, and E. Buckler, 2014 Entering the second century of maize quantitative genetics. Heredity 112: 30-38.

Wang, H., T. Nussbaum-Wagler, B. Li, Q. Zhao, Y. Vigouroux, et al., 2005 The origin of the naked grains of maize. Nature 436: 714-719.

Wang, M., Z. Chen, H. Zhang, H. Chen, and X. Gao, 2018 Transcriptome analysis provides insight into the molecular mechanisms underlying gametophyte factor 2-mediated cross-incompatibility in maize. Int J Mol Sci 19.

Whitt, S. R., L. M. Wilson, M. I. Tenaillon, B. S. Gaut, and E. S. Buckler, 2002 Genetic diversity and selection in the maize starch pathway. Proceedings of the National Academy of Sciences 99: 12959-12962.

Wickham, H., 2016 ggplot2: Elegant Graphics for Data Analysis. Springer-Verlag New York.

Wickham, H., R. François, L. Henry, and K. Müller, 2020 dplyr: A Grammar of Data Manipulation. R package version 1.0.2.

Wilke, C. O., 2020 cowplot: Streamlined Plot Theme and Plot Annotations for 'ggplot2'. R package version 1.1.0.

Wilkes, H. G., 1977 Hybridization of maize and teosinte, in mexico and guatemala and the improvement of maize. Economic Botany 31: 254-293.

Yeaman, S., 2013 Genomic rearrangements and the evolution of clusters of locally adaptive loci. Proceedings of the National Academy of Sciences 110: E1743-E1751.

Yost, J. M. and K. M. Kay, 2009 The evolution of postpollination reproductive isolation in costus. Sex Plant Reprod 22: 247-55.

Zigler, K., E. Raff, E. Popodi, R. Raff, and H. Lessios, 2003 Zigler $\mathrm{ks}$, raff ec, popodi e, raff ra, lessios ha. adaptive evolution of bindin in the genus heliocidaris is correlated with the shift to direct development. evolution 57: 2293-2302. Evolution 57: 2293-302. 
bioRxiv preprint doi: https://doi.org/10.1101/2020.11.10.377481; this version posted November 11,2020 . The copyright holder for this preprint (which was not certified by peer review) is the author/funder, who has granted bioRxiv a license to display the preprint in perpetuity. It is made available under aCC-BY-NC-ND 4.0 International license.
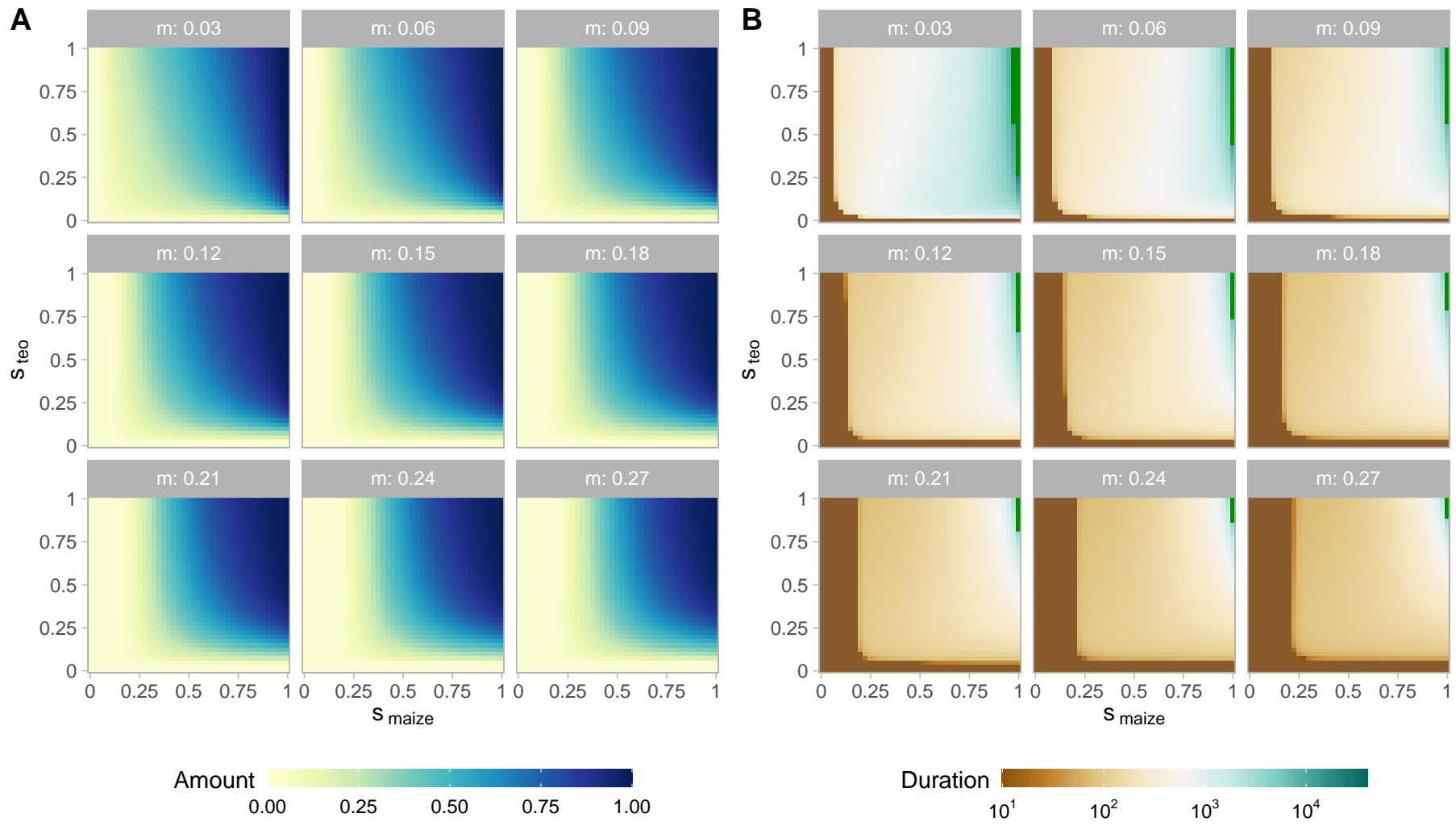

Figure S1 The impact of asymmetric selection on the extent (A) and duration (B) of reinforcement: Over a range of migration rates with $g_{\text {maize } \rightarrow \text { teo }}=g_{\text {teo } \rightarrow \text { maize }}=0.03$ and $r_{\mathcal{A M}}=r_{\mathcal{M F}}=10^{-4}$ 
bioRxiv preprint doi: https://doi.org/10.1101/2020.11.10.377481; this version posted November 11,2020 . The copyright holder for this preprint (which was not certified by peer review) is the author/funder, who has granted bioRxiv a license to display the preprint in perpetuity. It is made available under aCC-BY-NC-ND 4.0 International license.
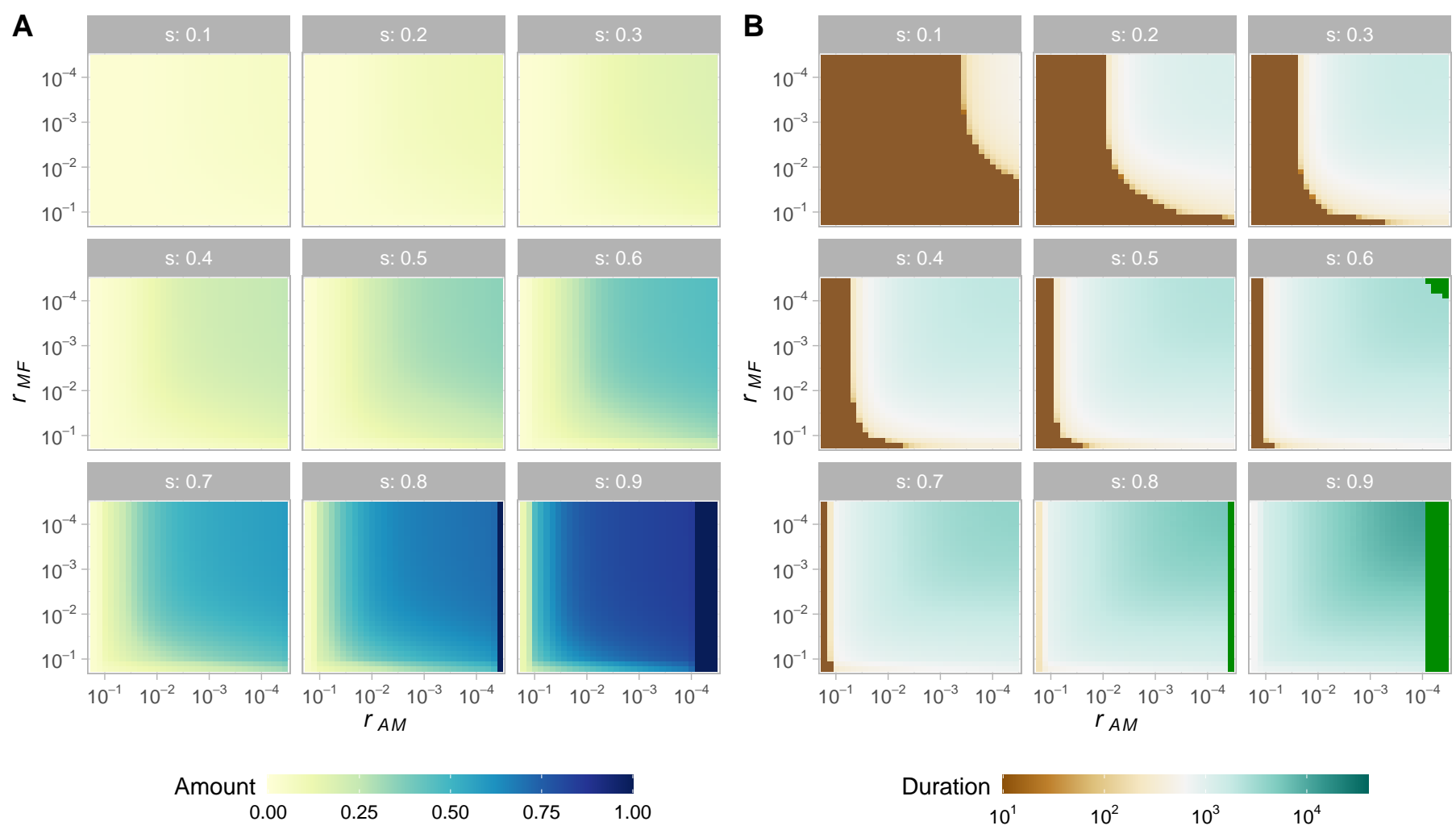

Figure S2 The impact of linkage on the extent (A) and duration (B) of reinforcement: Over a range of symmetric selection coefficients. Symmetric selection coefficient and $g_{\text {maize } \rightarrow \text { teo }}=g_{\text {teo } \rightarrow \text { maize }}=0.03$. We note that the $\mathrm{x}$-axis moves from loose linkage on the left to tight linkage on the right. 


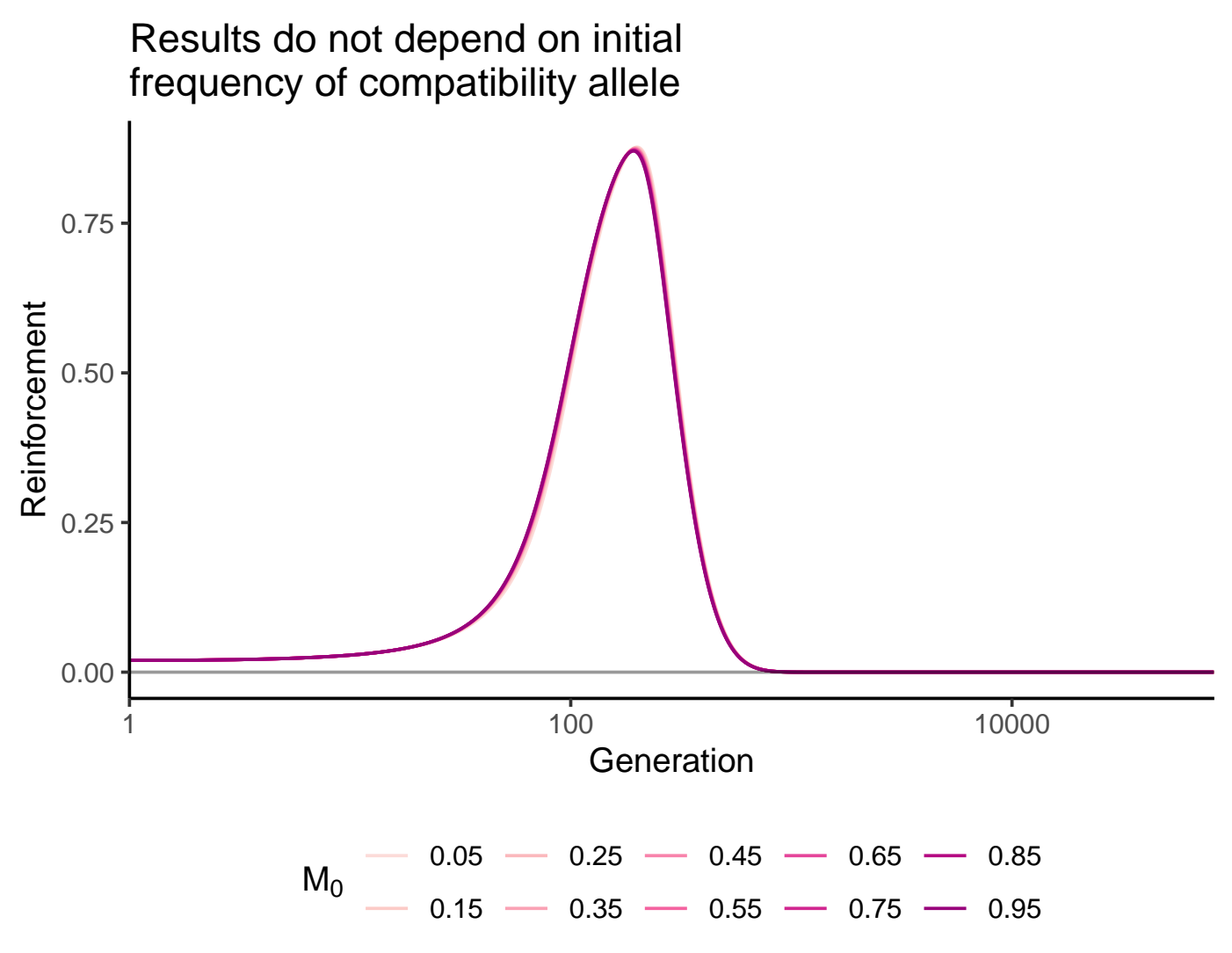

Figure S3 Results are robust to initial frequency of $M$ in teosinte. The impact of variability in the initial frequency of pollen compatibility allele, $M$, in teosinte on the transient reinforcement of postmating-prezygotic isolation (note all lines are on top of one another). Parameter values: Selection $-s_{\text {teo }}=s_{\text {maize }}=0.75$. Migration $-\left(g_{\text {maize } \rightarrow \text { teo }}=g_{\text {teo } \rightarrow \text { maize }}=0.1\right)$. Recombination $-r_{\mathcal{A M}}=$ $r_{\mathcal{M F}}=0.0001$. Allele frequencies $-f_{M 0 \text {,teo }}=$ displayed by color, $f_{M 0 \text {, maize }}=0, f_{F 0 \text {,teo }}=0.01, f_{F 0 \text {,maize }}=0$. 


\section{Results quantitatively depend on barrier strength}

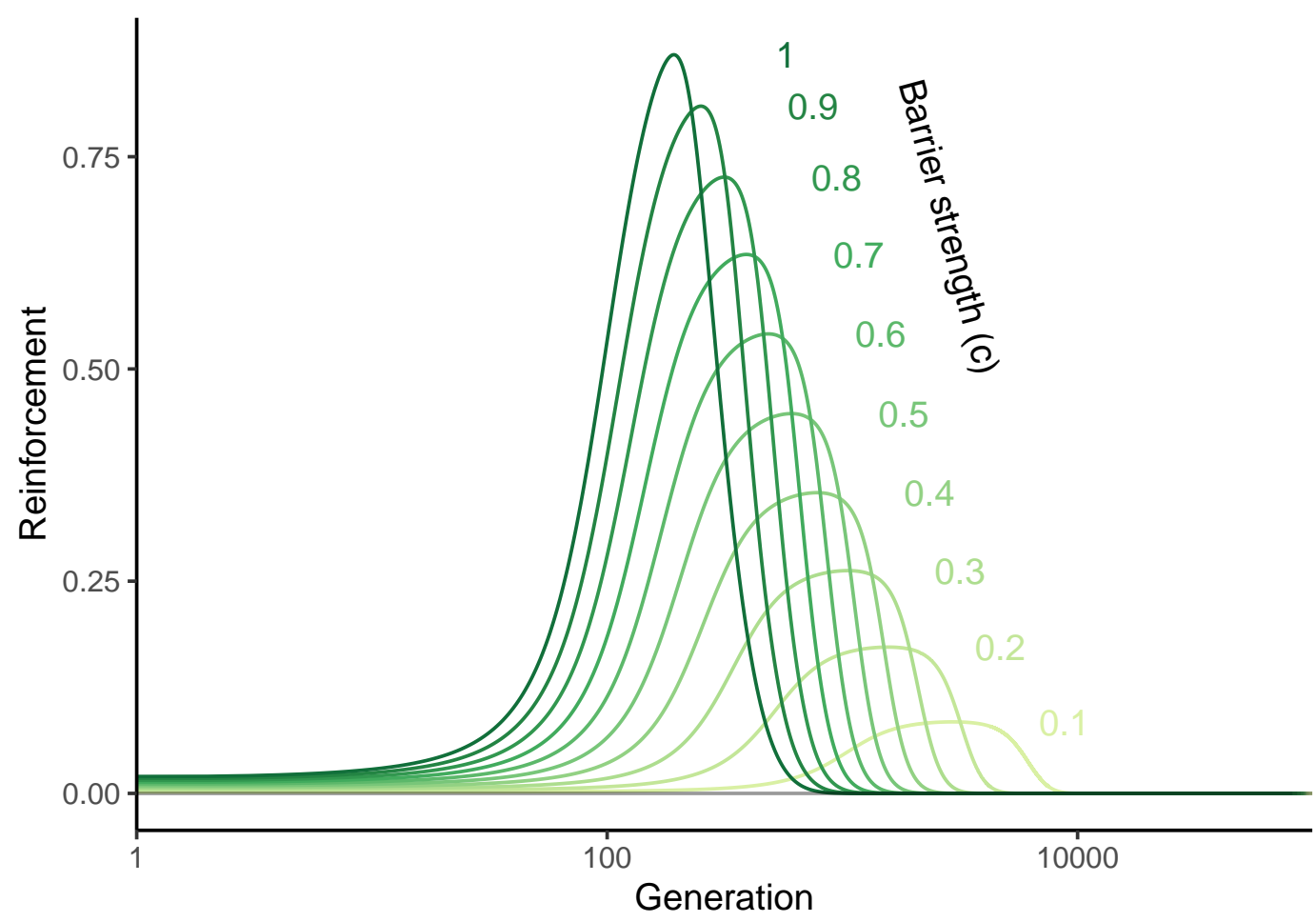

Figure S4 Results change quantitatively with imperfect barriers. We allow for an imperfect barrier by asserting that style with mating barrier genotypes are fertilized by a given haplotype, $k$, with probability $x_{k}=\frac{p_{k}\left(1-\delta_{k} c\right)}{\sum x_{k}}$, where $p_{k}$ is the frequency of haplotype $k$ in pollen after fertilization, $\delta_{k}$ equals zero for compatible pollen grains and one for incompatible pollen grains. $c$ the efficacy of the barrier is colored in the plot above. Parameter values: Selection $-s_{\text {teo }}=s_{\text {maize }}=0.75$. Migration $-(g$ maize $\rightarrow$ teo $=$ $g_{\text {teo } \rightarrow \text { maize }}=0.1$ ). Recombination $-r_{\mathcal{A M}}=r_{\mathcal{M F}}=0.0001$. Allele frequencies $-f_{M 0 \text {,teo }}=1, f_{M 0, \text { maize }}=0, f_{F 0, \text { teo }}=0.01$, $f_{F 0, \text { maize }}=0$. 